\title{
Adenoma adrenocortical ectópico parahiliar renal, en paciente con síndrome de Cushing independiente de ACTH. Abordaje laparoscópico. Reporte de caso y revisión de la literatura
}

\section{Adrenocortical ectopic adenoma in a patient with ACTH independent Cushing's syndrome. Laparoscopic approach. Case report and review of literature}

\author{
Carlos Eduardo Hernández ${ }^{1}$ José Gustavo Ramos Ulloa ${ }^{2}$ Sara Rocío Peralta Pizza ${ }^{3}$ Mauricio Moreno \\ 1 Urólogo, Unidad de Urología, Clínica Nogales, Bogotá, Colombia \\ 2 Urólogo, Pontificia Universidad Javeriana, Bogotá, Colombia \\ ${ }^{3}$ Médica y cirujana, Pontificia Universidad Javeriana, Bogotá, Colombia \\ Address for correspondence Sara Rocío Peralta Pizza, Médica y \\ cirujana, Pontificia Universidad Javeriana, Bogotá, Colombia \\ (e-mail: speraltamd@gmail.com).
}

${ }^{4}$ Urólogo, Unidad de Urología, Hospital de la Policía, Bogotá, Colombia

Urol Colomb 2018;27:181-186.

Resumen

Palabras clave

- síndrome de cushing

- coristoma

- adenoma corticosuprarrenal

- tumor de resto suprarrenal

- laparoscopía

\section{Abstract}

Objetivos El adenoma adrenocortical ectópico es el producto de la transformación de tejido adrenal ectópico embriológico en adenoma funcionante. Su asociación con síndrome de Cushing corresponde a menos del $2 \%$ de los casos. El presente es el noveno caso reportado en la literatura, por lo tanto se pretende recalcar la manera de realizar un adecuado abordaje diagnóstico multidisciplinario logrando el enfoque quirúrgico que permite el mejor tratamiento.

Materiales y métodos Búsqueda en bases de datos Pubmed, Lilacs, Embase. Revisión de la historia clínica y seguimiento postoperatorio.

Resultados Paciente femenina de 28 años quien presentó un aborto en primer trimestre de embarazo, asociado a hipertensión arterial, facies de luna llena y obesidad centrípeta. Se encuentra masa pararrenal izquierda. Se realiza abordaje laparoscópico para la resección de la misma, con patología que informa adenoma cortical adrenal. Resolución de sintomatología después del procedimiento.

Conclusiones Es un caso de baja frecuencia, donde es importante identificar la sintomatología temprana, así como realizar un abordaje multidisciplinario con el fin de realizar un correcto abordaje diagnóstico y terapéutico.

Objectives Ectopic adrenocortical adenoma is the result of the transformation of adrenal ectopic tissue in functional adenoma. It is associated with Cushing's syndrome is less than $2 \%$ of cases. The case presented here is the 9 th reported in literature. Emphasis is placed on a comprehensive multidisciplinary diagnostic approach with a surgical focus that leads to a better management. received

December 20, 2016

accepted

March 25, 2017

published online

January 10, 2018
DOI https://doi.org/

10.1016/j.uroco.2017.03.011.

ISSN 0120-789X.

eISSN 2027-0119.
Copyright ( $)$ 2018, Sociedad Colombiana License terms de Urología. Publicado por Thieme Revinter Publicações Ltda., Rio de Janeiro, Brazil. Todos los derechos reservados.

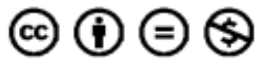




\section{Keywords}

- cushing's syndrome

- choristoma

- adrenocortical adenoma

- adrenal rest tumour

- laparoscopy
Materials and methods Review of literature in PubMed, Lilacs, and Embase. Review of the clinical records and post-surgical follow-up of the patient.

Results The patient is a 28 year-old female who had a spontaneous abortion in the first-trimester that was associated with high blood pressure, moon face and centripetal obesity. A left para-renal mass was found, and a laparoscopic resection was performed on the tumour. The histopathology showed that it was an adrenal cortical adenoma. The symptoms resolved after the procedure.

Conclusions Although this is a rare case, it is important to identify early symptoms, using a multidisciplinary approach in order to make a correct diagnosis and its early management.

\section{Introducción}

El síndrome de Cushing (SC) endógeno se caracteriza por la sobreproducción crónica de cortisol y está asociado con aumento en la morbimortalidad. Presenta un conjunto de manifestaciones clínicas ( - Tabla 1$)^{1}$ que pueden variar dependiendo del nivel de cortisol de cada paciente, siendo los signos más característicos de esta enfermedad la obesidad central con debilidad muscular proximal, hematomas, estrías violáceas mayores de $1 \mathrm{~cm}$ e hipertensión arterial. ${ }^{1,2}$ En niños la presentación clínica es diferente, siendo el aumento de peso y el retardo del crecimiento lo más frecuente. ${ }^{1}$ Se ha visto involucrado en el 2 al 3\% los casos de pacientes con

Tabla 1 Signos y síntomas de síndrome de Cushing

\begin{tabular}{|l|}
\hline Signos y síntomas \\
\hline La obesidad central con: \\
\hline Facie de luna llena con plétora \\
\hline El aumento de la grasa supraclavicular y dorso cervical \\
\hline Adelgazamiento de la piel con equimosis \\
\hline Amplias estrías violáceas (mayor de $1 \mathrm{~cm}$ ) \\
\hline Miopatía proximal \\
\hline Aumento de lanugo, hirsutismo \\
\hline Infecciones fúngicas superficiales \\
\hline Retraso del crecimiento (en niños) \\
\hline Trastornos psiquiátricos \\
\hline Diagnóstico clínico \\
\hline Síndrome metabólico $X$ \\
\hline La diabetes mellitus (A1C $>8 \% \mathrm{Hb})$ \\
\hline Hipertensión, hiperlipidemia \\
\hline Síndrome de ovario poliquístico (SOP) \\
\hline Hipogonadismo hipogonadotrópico \\
\hline Oligomenorrea/amenorrea/infertilidad \\
\hline Disminución de la libido e impotencia \\
\hline Osteoporosis (especialmente fractura de costilla) \\
\hline Pacientes < 65 años \\
\hline
\end{tabular}

Fuente: Findling et al. ${ }^{1}$ síndrome metabólico, principalmente diabetes mellitus e hipertensión arterial, que presentan pobre respuesta al tratamiento farmacológico ${ }^{1,01562}$ (-Tabla 2).

El SC se clasifica en ACTH-dependiente y ACTHindependiente, correspondiendo este último al 20\% de los casos. La etiología de SC ACTH-independiente corresponde en un $60 \%$ a adenomas suprarrenales y un $40 \%$ a carcinomas suprarrenales. ${ }^{3}$ En menos del $2 \%$ se encuentran otras causas, dentro de las cuales está el adenoma adrenocortical ectópico, encontrándose reportados 8 casos hasta el momento.

Se revisa este caso clínico dada la baja frecuencia de presentación, con el objetivo de analizar el abordaje diagnóstico multidisciplinario, el enfoque clínico y el acceso quirúrgico.

\section{Materiales y métodos}

Revisión de la literatura, búsqueda en las bases de datos Pubmed, Lilacs, Embase con las palabras clave "Choristoma» AND «Adrenocortical Adenoma» OR «Adrenal Gland» $\mathrm{y}$ «Ectopic adrenal Adenoma», se revisaron los diferentes artículos, encontrando en total 8 reportes de caso de adenoma corticoadrenal ectópico con presentación clínica de SC.

\section{Resultados}

Paciente femenina de 28 años quien presentó un aborto en el primer trimestre de embarazo asociado a hipertensión arterial de etiología no establecida. Progresivamente desarrolla facies de luna llena, obesidad centrípeta, caída del cabello y estriaciones violáceas en el abdomen. Se inició manejo con losartán y se realizaron estudios complementarios encontrando cortisol libre en orina en 386,6 UG/24 h. Endocrinología realiza diagnóstico de SC. Los estudios diagnósticos muestran cortisol sérico y cortisol libre en orina en 24 h, elevados; test de supresión con dexametasona, el cual no suprime; la medición de ACTH se encontraba menor de $5 \mathrm{pg} / \mathrm{ml}$, por lo cual se realizó el diagnóstico de SC independiente de ACTH. En el estudio imagenológico con TAC abdominopélvico contrastado se observó una masa pararrenal izquierda por debajo del hilio renal de $35 \times 40 \mathrm{~mm}$ (-Fig. 1). Se inició manejo médico con ketoconazol y se programó para procedimiento quirúrgico. Se realizó un abordaje laparoscópico encontrando una masa 
Tabla 2 Reportes de caso de SC asociado a adenoma adrenal ectópico pararrenal

\begin{tabular}{|l|l|l|l|l|l|}
\hline Caso & Autor & $\begin{array}{l}\text { Fecha de } \\
\text { publicación }\end{array}$ & Sexo/edad & Diámetro $>\mathbf{( c m})$ & Localización \\
\hline 1 & Chaffee $\mathrm{WR}^{7}$ & 1963 & $\mathrm{M} / 40$ & - & $\begin{array}{l}\text { Posteromedial a polo } \\
\text { renal izquierdo }\end{array}$ \\
\hline 2 & Leibowitz J & 1998 & $\mathrm{~F} / 33$ & 3,0 & $\begin{array}{l}\text { Proximal a suprarrenal } \\
\text { izquierda }\end{array}$ \\
\hline 3 & Ayala $\mathrm{AR}^{9}$ & 2000 & $\mathrm{~F} / 63$ & 3,5 & Hilio renal izquierdo \\
\hline 4 & Louiset $\mathrm{E}^{10}$ & 2010 & $\mathrm{~F} / 35$ & 3,8 & Pararrenal izquierdo \\
\hline 5 & Wang $\mathrm{XL}^{11}$ & 2012 & $\mathrm{M} / 38$ & 5,3 & Hilio renal izquierdo \\
\hline 6 & Cardinalli IA & & & Hilio renal izquierdo \\
\hline 7 & Tong $\mathrm{A}^{13}$ & 2012 & $\mathrm{~F} / 17 \mathrm{~m}$ & 1,1 & Hilio renal izquierdo \\
\hline 8 & Zhang J & $\mathrm{F} / 53$ & 3,5 & Seno renal derecho \\
\hline 9 & Caso actual & 2014 & $\mathrm{~F} / 37$ & 3,4 & Inferior a vena renal izquierda \\
\hline
\end{tabular}

pararrenal debajo de la vena renal con compresión de la vena gonadal izquierda, en relación con un doble sistema colector completo ( $\mathbf{- F i g . ~ 2 ) . ~ L a ~ p a t o l o g i ́ a ~ m u e s t r a ~ u n a ~ m a s a ~ d e ~}$ $4 \times 3 \times 1,5 \mathrm{~cm}$ homogénea; el estudio de inmunohistoquímica (-Fig. 3) presenta marcadores positivos para S100, MELAN-A, sinaptofisina, inhibina y HMB45 y negativos para AE1/AE23, CAM 5,2; cromogranina, PAX8 y RCC, lo cual confirma el diagnóstico de adenoma cortical adrenal. Durante el manejo y evolución postoperatoria la paciente presenta una notoria mejoría de su

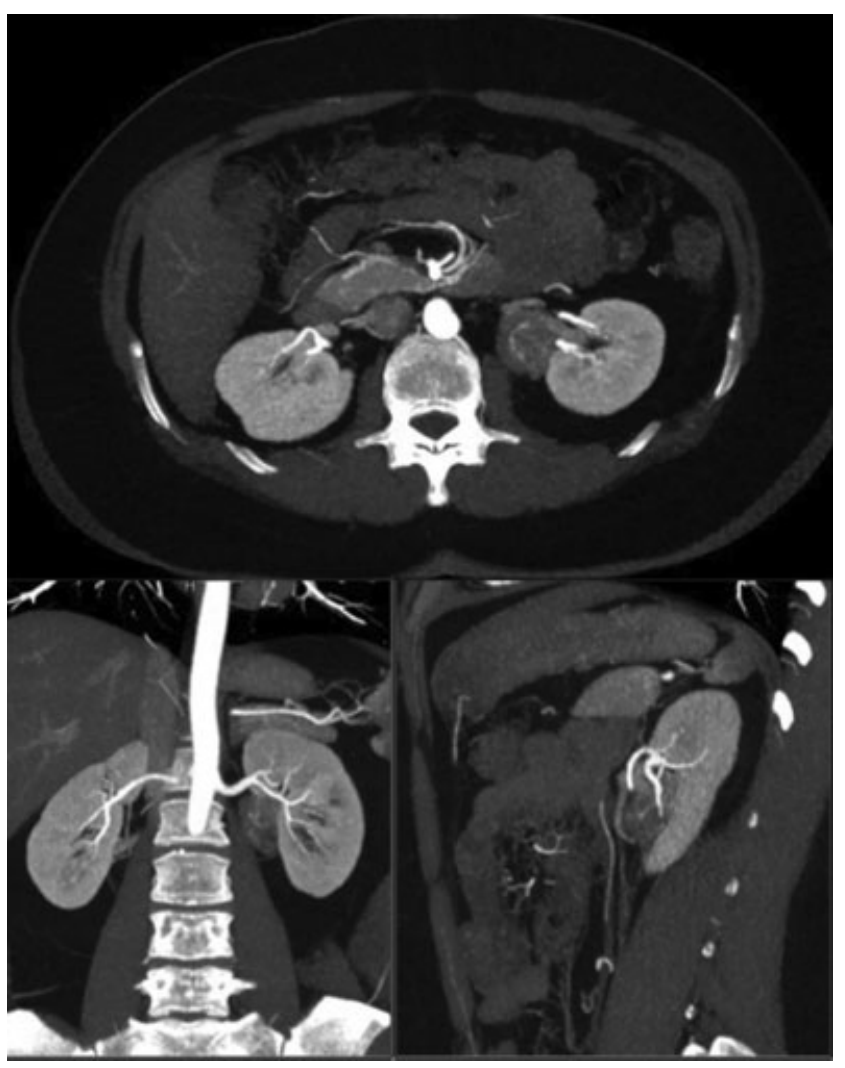

Fig. 1 Angio-TAC abdominal: masa pararrenal izquierda inferior al hilio renal de $35 \times 40 \mathrm{~mm}$. sintomatología y se observa una adecuada regulación de los niveles de cortisol.

\section{Discusión}

El SC constituye un variado conjunto de manifestaciones con un origen que puede ser tan amplio como un trastorno en la regulación de la producción de cortisol, o una anomalía en la migración del tejido corticoadrenal. Por lo que requiere un enfoque claro y preciso.

El abordaje diagnóstico bioquímico (-Fig. 4$)^{3}$ en estos pacientes se realiza inicialmente mediante la medición de cortisol urinario libre en $24 \mathrm{~h}$, el test de supresión con dosis baja de dexametasona o la medición de cortisol sérico o salival a las 23-24 h. En general se recomienda realizar 2 pruebas para confirmar el diagnóstico, sin embargo cuando la clínica es típica con un examen positivo se puede confirmar el diagnóstico. Posteriormente se realiza la medición de ACTH, la cual si se encuentra menor de $5 \mathrm{pg} / \mathrm{ml}$ significa que se trata de un SC ACTH-independiente y la posible etiología es probablemente adrenal, por lo cual es indicado realizar una imagen para explorar las glándulas suprarrenales ${ }^{3}$ que en nuestro caso nos permitió observar la masa parahiliar renal izquierda que correspondió con un adenoma adrenal ectópico.

El adenoma adrenocortical ectópico se considera producto de la transformación de tejido adrenal ectópico en adenoma funcionante. Estos remanentes aberrantes de tejido adrenal se pueden encontrar a lo largo de la ruta de migración que realizan las glándulas suprarrenales durante el periodo embriológico.

Dentro de las localizaciones más frecuentes del tejido corticoadrenal ectópico se encuentran: plexo celiaco, $32 \%$; ligamento ancho, 23\%; anexo al testículo, 7,5\%; riñón 0,1-0,6\% y cordón espermático 3,8-9,3\%, ${ }^{4}$ sin embargo se han descrito otras localizaciones menos comunes como son pared gástrica, apéndice, hígado, pulmones, cerebro. Su incidencia en autopsias depende de la edad, encontrándose en la edad pediátrica hasta en un $50 \%$, y en adultos solo en un $1 \%$, debido a atrofia de este tejido con la edad. ${ }^{4,5}$ En general el tejido corticoadrenal ectópico no es funcional, siendo su hallazgo incidental durante otros 


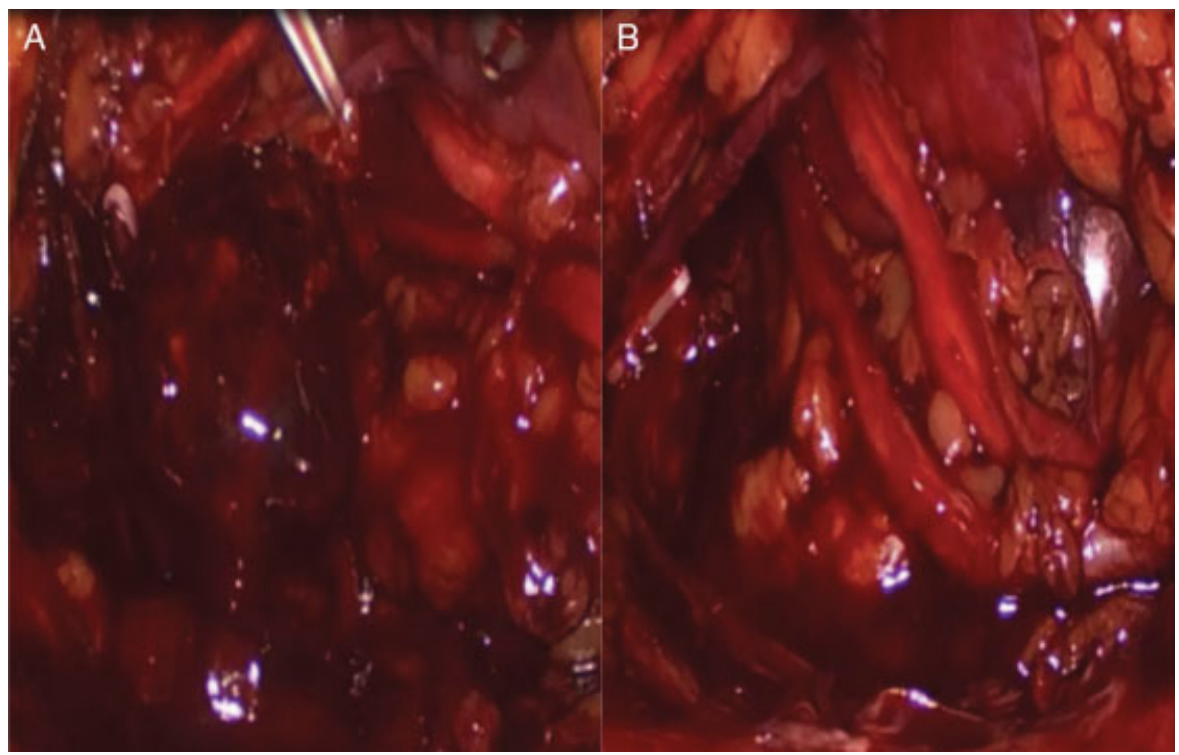

Fig. 2 Laparoscopia. A) Adenoma adrenal ectópico. B) Doble sistema colector completo.

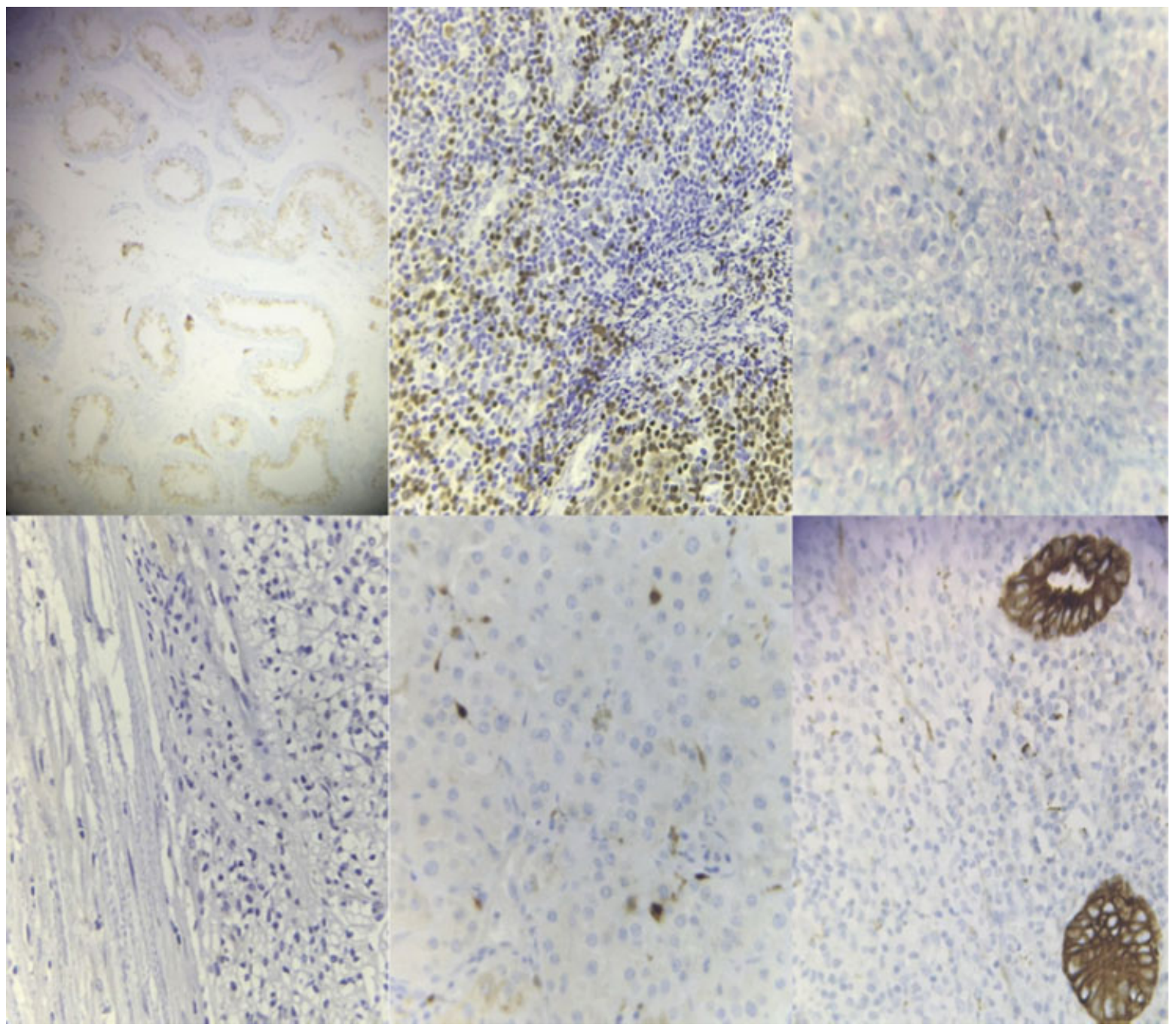

Fig. 3 Inmunohistoquímica. 


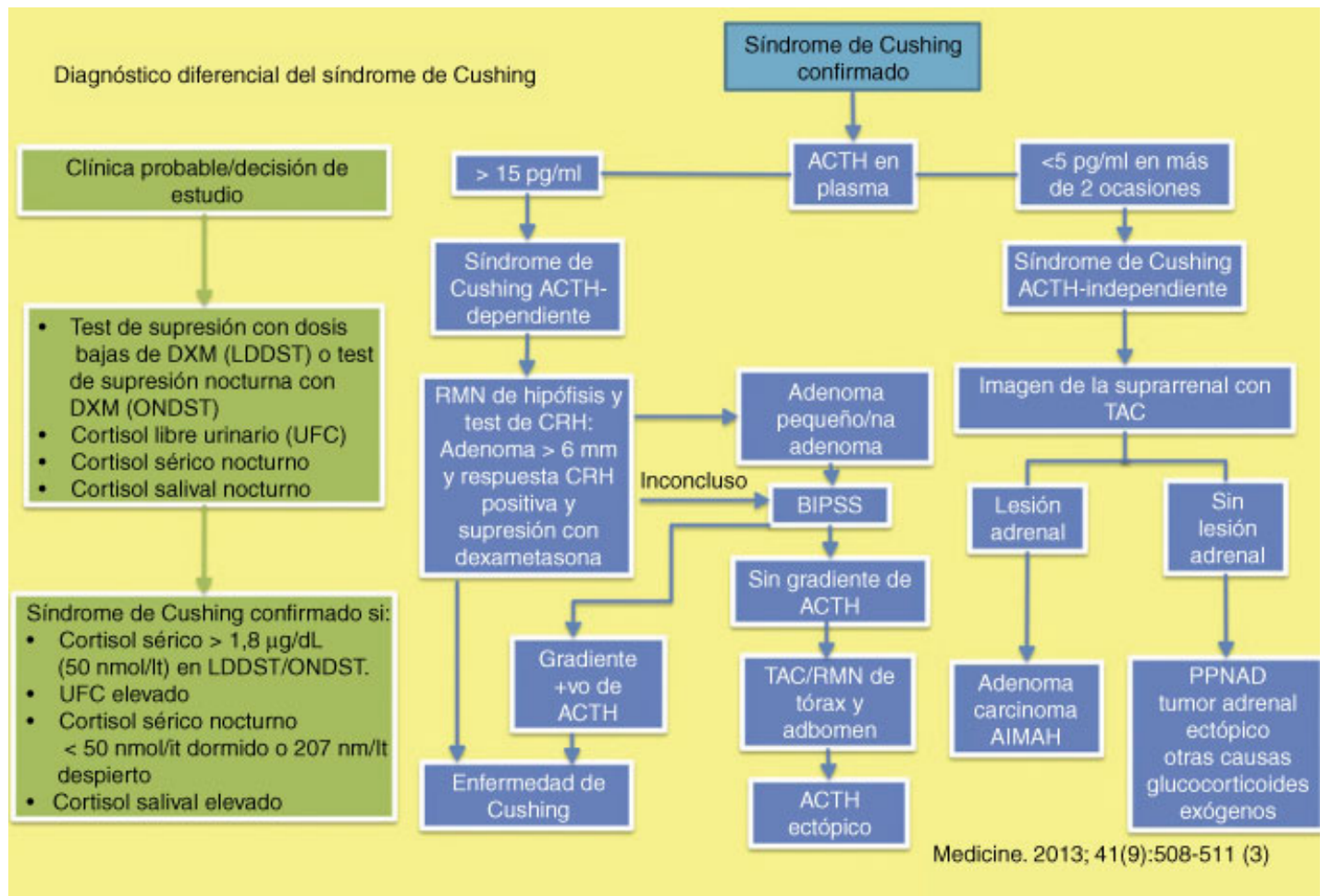

Fig. 4 Diagnóstico bioquímico del síndrome de Cushing. AIMAH: hiperplasia adrenal macronodular ACTH-independiente; BIPSS: muestreo de senos petrosos inferiores bilateral; PPNAD: enfermedad adrenocortical nodular pigmentada primaria.

procedimientos quirúrgicos, sin embargo en algunos casos puede presentar producción hormonal y en estos casos el diagnóstico se hace en el estudio de la sintomatología.

El tratamiento de los pacientes con tumores ectópicos adrenales es quirúrgico en los casos de sospecha de malignidad y si presentan producción hormonal. Por lo tanto, el tratamiento en el SC por adenoma suprarrenal ectópico es fundamentalmente quirúrgico, siendo el manejo médico únicamente indicado en los casos en los que se encuentre contraindicada la cirugía o como terapia puente en espera del procedimiento quirúrgico. ${ }^{6}$ La vía de abordaje del paciente dependerá de la experiencia del cirujano y de la situación específica de cada paciente. Si se logra realizar la extracción completa del tumor, se obtiene una mejoría temprana de los síntomas y en general un pronóstico favorable, como se ha visto también en todos los demás casos reportados similares al nuestro.

\section{Conclusión}

Se trata de un adenoma adrenocortical ectópico parahiliar renal, clínicamente manifiesto por un SC independiente de ACTH. Se trata del noveno caso reportado en la literatura. Es importante identificar la sintomatología temprana, así como realizar un abordaje multidisciplinario con el fin de realizar un correcto abordaje diagnóstico y terapéutico.

\section{Responsabilidades éticas}

\section{Protección de personas y animales}

Los autores declaran que los procedimientos seguidos se conformaron a las normas éticas del comité de experimentación humana responsable y de acuerdo con la Asociación Médica Mundial y la Declaración de Helsinki.

\section{Confidencialidad de los datos}

Los autores declaran que han seguido los protocolos de su centro de trabajo sobre la publicación de datos de pacientes.

\section{Derecho a la privacidad y consentimiento informado}

Los autores han obtenido el consentimiento informado de los pacientes y/o sujetos referidos en el artículo. Este documento obra en poder del autor de correspondencia.

Conflicto de intereses

Los autores declaran no tener ningún conflicto de intereses.

\section{Bibliografía}

1 Findling JW, Raff H. Screening and diagnosis of Cushing's syndrome. Endocrinol Metab Clin North Am 2005;34:385-402

2 Newell-Price J. Diagnosis/differential diagnosis of Cushing's syndrome: a review of best practice. Best Pract Res Clin Endocrinol Metab (Suppl 12009;23:S5-S14

3 Daniel E, Newell-Price J. Cushing's Syndrome. MEDICINE 2013; 41:508-511

4 Puga Bermúdez R, Ochoa Mejias R, Ramia Ángel JM, Pérez Mies B García-Parreño Jofré J. Tejido corticoadrenal ectópico de localización pelviana. Cirugía Española 2011;89:688-689

5 Anderson JR, Ross AH. Ectopic adrenal tissue in adults. Postgr Med J 1980;56:806-808

6 Creemers SG, Hofland LJ, Lamberts SW, Feelders RA. Cushing's syndrome: an update on current pharmacotherapy and future directions. Expert Opin Pharmacother 2015;16:1829-1844 
7 Chaffee WR, Moses AM, Lloyd CW, Rogers LS, Soffer LJ, Graham LS, et al. Cushing's syndrome with accessory adrenocortical tissue. JAMA J Am Med Assoc 1963;186:799

8 Leibowitz J, Pertsemlidis D, Gabrilove JL. Recurrent Cushing's syndrome due to recurrent adrenocortical tumor-fragmentation or tumor in ectopic adrenal tissue? J Clin Endocrinol Metab 1998; 83:3786-3789

9 Ayala AR, Basaria S, Udelsman R, Westra WH, Wand GS. Corticotropinindependent Cushing's syndrome caused by an ectopic adrenal adenoma. J Clin Endocrinol Metab 2000;85:2903-2906

10 Louiset E, Gobet F, Libé R, Horvath A, Renouf S, Cariou J, et al. ACTH-independent Cushing's syndrome with bilateral micronodular adrenal hyperplasia and ectopic adrenocortical adenoma. J Clin Endocrinol Metab 2010;95:18-24
11 Wang X-L, Dou J-T, Gao J-P, Zhong W-W, Jin D, Hui L, et al. Laparoscope resection of ectopic corticosteroid-secreting adrenal adenoma. Neuro Endocrinol Lett 2012;33:265-267

12 Cardinalli IA, de Oliveira-Filho AG. A unique case of synchronous functional adrenocortical adenoma and myelolipoma within the ectopic adrenal cortex in a child with Beckwith-Wiedemann syndrome. Pathol Res Pract 2012;208:189-194

13 Tong A, Jia A, Yan S, Zhang Y, Xie Y, Liu G. Ectopic cortisol-producing adrenocortical adenoma in the renal hilum: Histopathological features and steroidogenic enzyme profile. Int J Clin Exp Pathol 2014;7:4415-4421

14 Zhang J, Liu B, Song N, Lv Q, Wang Z, Gu M. An ectopic adreocortical adenoma of the renal sinus: a case report and literature review. BMC Urol 2016;16:3 